\title{
Comerse las Indias. La alimentación como clave clasificatoria del Nuevo Mundo en la obra de Fernández de Oviedo*
}

\author{
To Eat the New World. Food as the Code to Organize \\ the Americas in the Work of Fernández de Oviedo
}

Daniel Egaña Rojas

Universidad de Chile

Aunque la descripción de la naturaleza del Nuevo Mundo en la obra de Gonzalo Fernández de Oviedo ha sido estudiada como la precursora de la historia natural indiana, una lectura detallada evidencia otras genealogías posibles. El artículo plantea que, antes que una historia natural, la naturaleza es vista y clasificada por Oviedo a partir de un criterio alimentario, intentando volver a situar las Indias en la ruta de las especias, de la que habian sido apartadas a fines del siglo $X V$.

Palabras Claves: Fernández de Oviedo; Nuevo Mundo; Naturaleza; Clasificación; Alimentación.

The description of nature in Gonzalo Fernández de Oviedo's work has been studied as the beginning of the research on the natural history of the New World, which a closer reading makes it possible to link to other disciplinary fields. In this article, it is suggested that Oviedo was approaching its nature with the aim of placing the New Continent within the context of the spice trade, from where it had been removed at the end of the $15{ }^{\text {th }}$ century.

Keywords: Fernández de Oviedo; New World; Nature; Classification; Food.

* N. de R.: Trabajo basado en la tesis doctoral del autor. Egaña, 2013, 258-276. 
Gracias a su edición de 1851 a cargo de José Amador de los Ríos y probablemente su reimpresión paraguaya en 1945, la obra indiana de Gonzalo Fernández de Oviedo ha sido profusamente estudiada. ${ }^{1}$ Ya en los tempranos trabajos de Enrique Álvarez, ${ }^{2}$ esta fue puesta en valor desde la perspectiva de la historia natural. Diversos autores han remarcado la importancia de sus escritos como el primer trabajo serio por dar cuenta de la naturaleza del Nuevo Mundo. ${ }^{3}$ Aunque su aproximación no siempre es sistemática, se suele destacar la acuciosidad y detalle con que Fernández de Oviedo describe la flora y fauna del Nuevo Mundo.

Si bien su obra como precursora de cierta historia natural indiana cuenta con una aceptación generalizada, ${ }^{4}$ el presente artículo plantea revisitar esta dimensión. Junto a la tradición que lo ubica al inicio de una historia natural del Nuevo Mundo, su descripción de la flora y la fauna lo hacen partícipe de otra genealogía, menos hegemónica pero no carente de centralidad para la cultura americana. Como pretendemos mostrar, bajo su descripción de la naturaleza indiana, que lo vincula en la historia natural, opera un segundo criterio clasificatorio que permite reinscribir esta dimensión de su obra en una nueva clase de saber, este es, el culinario.

Parte de una obra mayor, la preocupación por lo culinario participa del intento de Fernández de Oviedo por entregar una descripción completa del Nuevo Mundo que guíe la expansión colonial, es decir, al mismo tiempo que traduce la novedad, atraiga a población europea y genere un sentido sobre la vida cotidiana del mundo indiano.

\section{Ordenar el mare magno}

Desde los primeros contactos con el Nuevo Mundo, los europeos no pudieron dejar de comentar la flora y fauna indiana. Sobre todo la flora fue catalogada desde un principio como exuberante, pero también como «disforme», «desemejante» y desconocida. ${ }^{5}$ Esta idea, recurrente entre los primeros observadores europeos, va a ser conceptualizada por Fernández de Oviedo bajo la idea del mare magno. Para él muchos de los árboles del Nuevo Mundo

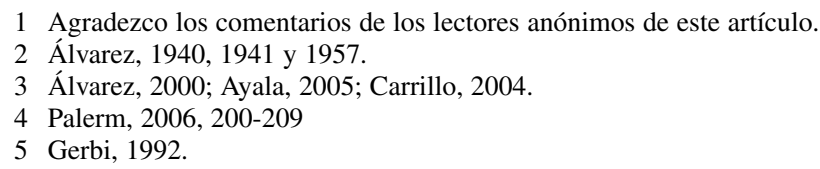


ni los indios naturales los conocen, ni saben dar nombre a la mayor parte dellos, ni los cristianos mucho menos, por serles cosa tan nueva e no conocida ni vista por ellos antes. [...] Y lo que en esto se podría decir es un mare magno e oculto; porque, aunque se ve, lo más dello se inora, porque no se saben, como he dicho, los nombres de tales árboles, ni sus propiedades. ${ }^{6}$

Darles un nombre y asignarles propiedades, son dos gestos clasificatorios por excelencia. Sin embargo, estos nunca ocurren en el vacío. En el caso de Fernández de Oviedo, la operación se enmarca en al menos dos tradiciones. Por una parte, como él mismo se encarga de explicitar en su obra indiana, por la referencia y prolongación de la obra de Plinio el Viejo, Naturalis Historia. En su razonamiento, si el Nuevo Mundo representa una parte del orbe ignota para la conciencia clásica, la obra de Plinio debe ser complementada con la descripción de la nueva naturaleza. Así, Plinio -y el mundo clásico en general — va a constituirse como un referente ineludible con el cual se compare, contraste y discuta la naturaleza del Nuevo Mundo. Pero esto solo es posible en el marco de la segunda tradición que confluye en su operación clasificatoria, a saber, la episteme medieval marcada por la semejanza. Como ha destacado Foucault,

hasta fines del siglo XVI la semejanza ha desempeñado un papel constructivo en el saber de la cultura occidental. En gran parte, fue ella la que guio la exégesis en interpretación de los textos; la que organizó el juego de los símbolos, permitió el conocimiento de las cosas visibles e invisibles, dirigió el arte de representarlas. El mundo se enrollaba sobre sí mismo: la tierra repetía el cielo, los rostros se reflejaban en las estrellas y las hierbas ocultaban en sus tallos los secretos que servían a los hombres. ${ }^{7}$

Así, en un principio, la clasificación operará por un doble gesto: prolongación de las categorías de Plinio e identificación por semejanza de la naturaleza indina. ${ }^{8}$ Sobre estas dos, sin embargo, Fernández de Oviedo

6 Fernández de Oviedo, lib. IX, Proemio, 1992, 1, 277.

7 Foucault, 2003, 26.

8 Probablemente, el caso límite de la identificación por semejanza de la naturaleza del Nuevo Mundo ocurre cuando Fernández de Oviedo realiza el inventario de las hierbas encontradas en la isla de La Española, las cuales asimila al herbolario medieval: «hay chicorias, o cicoria; cerrajas que llaman los herbolarios rostrum porcinum; verdolagas o pertulaca; verbena o verbena; hierbamora o solatrum; llantén, al cual los médicos llaman plantago; pan y quesillo, alias bursa pastoris; altamisa, alias matriarcaria; escudete, alias nenúfar; albahaca u ozimum gariophilatum, alias basilipo; lengua cerval o scoloprendia; culantrillo de pozo o capilus Veneris; poleo o politrique politricum; doradilla o ceteraque; deiantos o adiantos; poleo montesino, poligium agreste; persicaria o herva maculata; malvavisco o altea; polipodio o polipodium; muérdago de roble, aunque nace acá sobre otros árboles, o viscus querci; abrojos de mar o tribulus marinus. Bledos o bletus; salvia o lilifagus; granos de amor o milium solis; juncia redonda o ciperus; trébol hidiondo o trifolium leporinum. Todas estas hierbas hay acá, segund lo he entendido de nuestros boticarios e herbolarios e yo he visto las más dellas en estas Indias». Fernández de Oviedo, lib. XI, cap. II, 1992, 2, 18. 
introducirá un tercer criterio, a saber, su propia autoridad en tanto descripción directa de la naturaleza, en tanto testigo presencial y privilegiado del Nuevo Mundo; testigo ocular que permite ponderar, discutir y dirimir la distancia entre la tradición y la nueva experiencia.?

Si la naturaleza indiana se presenta como un «mare magno e oculto», la exploración que Fernández de Oviedo hace de ella adquiere, a momentos, la forma de un compendio, el cual busca guiar al lector europeo entre esta desconocida espesura. Carente de un sentido taxonómico moderno (post linneano), la organización de la flora va intentar replicar en el Nuevo Mundo la división propuesta por Plinio. De este modo, en un primer momento, Fernández de Oviedo divide la flora en categorías que, si bien no son excluyentes, intentan abarcar la diversidad indiana: de un lado están árboles frutales, tanto autóctonos como los traídos del Viejo Mundo, luego están los árboles y hierbas medicinales y, por último, aquellos que Oviedo llama árboles salvajes, entendiendo por estos últimos aquellos que no son cultivados y cuya utilidad, si no se conoce, debe desentrañarse.

Es en estos últimos que la doble tradición opera con más fuerza. Al no poseer fruto distintivo que pueda homologar a su saber europeo, basará el ejercicio comparativo en las autoridades clásicas. Con un gesto siempre imperfecto, Fernández de Oviedo busca señales que le permitan identificar los árboles indianos y así catalogarlos en el repertorio de la tradición. El problema es que difícilmente las señales que identifican a los árboles del Viejo Mundo se encuentran en su totalidad en un mismo espécimen de indiano. Por ejemplo, tras remitir a la descripción que hace Plinio sobre el terebinto, Fernández de Oviedo plantea que

es cierto que yo he muchas veces ocupándome inquiriendo este árbol (por mi persona), hallándome por estos caminos e boscajes en diversas partes destas Indias, y el que concierta en una señal, se desacuerda en otras. E así, por una sola que ven los que no tienen experiencia en las cosas, le conceden el nombre, así como si tuviera todas las artes e circunstancias que Plinio dice; pero yo he visto que estos mosquitos e otros los producen, o salen acá, de algunos árboles; e de otros salen mariposas; e de otros, cocos o gorgojos e otros animalejos de diversas maneras en sí; e también diversos árboles crían los mesmos animales. Estos terebintos de acá o cualquier árbol que ellos sean o así se llamen, no tienen mayor auctoridad de haberlos llamado así quien le pulgó. ${ }^{10}$

9 Brokaw, 2005, 143-65. Sobre este punto la crítica ha puesto especial énfasis. La idea de que Fernández de Oviedo legitima su relato como testigo ocular, ver Myers, 1990 y 2007, 137 y ss. Sobre el valor de la experiencia frente la tradición y la experiencia como método de conocer la naturaleza indiana, ver Baraibar, 2011, 21 y 2014, 16.

10 Fernández de Oviedo, lib. IX, cap. X, 1992, 1, 288. 
Al igual que con el terebinto, Fernández de Oviedo suele dudar de la identidad que los españoles - haciendo mimesis de la tradición eurocéntrica - le han dado a otros árboles indianos, como el cedro y el taray. Pero a falta de otra signatura, no tiene más alternativa que clasificarlos bajo esos nombres, mostrando su disconformidad. Ahora bien, esta cauta actitud crítica no siempre acompaña a Oviedo cuando revisa la flora indiana, encontrando - por ejemplo - entre los árboles salvajes que describe a pinos sin piñas, que si bien «en todo son perfectos pinos los de acá; pero no tan altos, ni tan gruesos ni tan derechos como los de tierra de Cuenca» $;{ }^{11}$ o a nogales, cuyo único inconveniente es «que las nueces destos de acá no son perfectas ni despiden la fructa, ni se pueden comer». ${ }^{12}$

Siguiendo la premisa del mare magno, es decir, de la utilidad oculta de lo provisto por la naturaleza indiana, Fernández de Oviedo intentará asignar una función a cada árbol salvaje. Desde las más tradicionales, como las del espino, que da buena madera para hacer sillas, fustes de sillas de montar, puertas y ventanas; hasta las más exóticas, como el caso del árbol de las cuentas de jabón, cuyas semillas sirven para lavar la ropa. A la mayor parte de los árboles salvajes, Fernández de Oviedo les atribuye funciones constructivas o de carpintería (mangle, roble, caobán, ceiba, cuya, maría, ciguas), mientras otros como el brasil, capera, nanci o el guao, que no poseen buena madera, sirven para hacer tintas y tinturas. ${ }^{13}$

Junto con una descripción que busca destacar la utilidad colonial del Nuevo Mundo, de algún modo, dar utilidad a la flora salvaje es también una estrategia para reforzar o restituir el imaginario de especiería: si bien el viaje colombino no llegó a Asia, las Indias occidentales ofrecen para Fernández de Oviedo la posibilidad de que su propia naturaleza sea explotada en la clave de las especias..$^{14}$ De este modo, destaca el fruto de los

11 Ibidem, lib. IX, cap. II, 279.

12 Ibidem, lib. IX, cap. III.

13 Esta actitud de describir, delimitar 7-34y buscar una utilidad a la naturaleza del Nuevo Mundo ha sido interpretado como un gesto ideológico por incorporar lo exótico y desconocido al interior de un ordenamiento imperial, gobernado por un soberano universal y cristiano. Ver Coello de la Rosa, 2002b. Si bien nuestra lectura no es incompatible con esta propuesta, indaga en un contenido específico de la descripción que realiza Fernández de Oviedo.

14 Es difícil establecer una sola definición sobre qué son las especies para el siglo XVI. Al igual que en la actualidad, estas cumplen una función de condimento, es decir, buscan sazonar los alimentos. Sin embargo, ello no se limita a un problema de hedonismo, ni tampoco remite a la idea comúnmente citada de "disfrazar" el mal sabor de la descomposición de algunos alimentos. Para el siglo XVI, las especies participan en la dietética de todo un imaginario galénico sobre la salud, humores, temperamentos, equilibrios y correspondencias entre lo que se come y quien lo come. Así, más que un alimento específico, semillas, frutos, raíces, cortezas e inclusos alimentos procesados como el 
agoreros, que poseen buen sabor y olor a almizcle, así como otro árbol que — asegura - huele a hinojo; en esta misma línea, las Indias poseerían su propio árbol de la canela, que si bien no es la misma que se conoce en el Viejo Mundo, su color y sabor son semejantes:

hay una buena canela, e de aquésta se trujo una carga o dos, por mandato de Atabaliba, de hacia la provincia de Quito, y es de otra forma que la canela de especiería, porque ésta es como vasillos o engaste de alguna fructa. ${ }^{15}$

Reinscribir las Indias en el horizonte de especiería implica dar cuenta de todas las potencialidades que la naturaleza en el Nuevo Mundo ofrece. Así, la vegetación indiana promete nuevas plantas que desbordan en usos prácticos, como es el caso de la palma. De su fibra se hacen telas y con su madera se fabrican saetas, vítores, lanzas jinetas, picas, macanas, e incluso instrumentos musicales. Pero lo más importante es su fruto, el cual considera de buen sabor, dulce y de carne espesa. Estos, llamados cocos porque — según explica Fernández de Oviedo— al tener tres agujeros parecen un mono «cocando», poseen diversas propiedades: se hace aceite, vino, y se lo come cocido, pues su sabor es mejor que el de las almendras; además posee una leche, la cual los cristianos comen con maíz.

Si bien la palma es un caso ejemplar de la sobre-utilidad que presenta la flora salvaje indiana, es en los árboles medicinales donde aparece con mayor persistencia la restitución del imaginario de especierías. La misma categoría clasificatoria — una botánica medicinal, ya presente en Pliniopretende establecer un valor agregado sobre la flora, al ligarla a un conocimiento útil para el ordenamiento colonial indiano. Así, Fernández de Oviedo establece una proto farmacopea donde incluye el árbol que suelda las quebraduras para curar las roturas de hueso, el guayacán para tratar las búas (sífilis), y el árbol del bálsamo, que cura heridas y mitiga dolores. Asimismo, incluye algunas hierbas como la llamada «y» que considera un purgante seguro, el goaconax, del que se hace un bálsamo para las heridas, el coigaraca que su usa para quitar la carne mala de las heridas, así como el perebecenuc y la perorica, dos hierbas que curan las llagas. ${ }^{16}$

azúcar, cumplen en la mayoría de los casos una función digestiva y medicinal que los constituyen como especias. Tanto su escasez como su especificidad en una salud estrictamente distribuida en clases sociales, hacen comprensible su alto valor social y económico que impulsa —al menos a nivel discursivola búsqueda de una especiería en el Nuevo Mundo. Ver Flandrin, 2011.

15 Fernández de Oviedo, lib. IX, cap. XXXI, 1992, 5, 92.

16 Fernández de Oviedo, lib. X, caps. I-VIII principalmente, 1992, 2, 8-20. 
Fernández de Oviedo no solo describe las plantas, sino que también entrega recetas y establece procedimientos para determinar las propiedades medicinales, dándole especial importancia a la experimentación:

todo lo que es medicinal requiere mucha experiencia, en especial en las cosas que nuevamente vienen a noticia de los hombre e que son poco usadas. [...] E así soy de la opinión que en este que llaman bálsamo (e no lo es sino algún licor bueno) que falta mucha parte de la experiencia a los que con él han de curar, e aquesta se ha de aprender también acaso, porque en dar más o menos en la cantidad, o en la calidad con que topa donde ha de obrar, podrá hacer lo que hacen las manzanillas con que se purgan algunos en estas partes, que a unos hacen provechos, e a otros mucho daño. ${ }^{17}$

En este sentido, Fernández de Oviedo reconoce la importancia de rescatar el saber medicinal prehispánico, como en el caso de la manzanilla de las avellanas — recién mencionada — donde el conocimiento de sus propiedades purgantes es aprendido mediante una indígena. En contra de lo que plantearía la imagen hegemónica en la historiografía sobre la vida de Fernández de Oviedo, este se lamenta de que el exterminio de la población nativa repercuta en el olvido de dicho saber:

como los indios antiguos son ya muertos, así se ha acabado con ellos el conocimiento que por su aviso se pudiera haber de propiedades semejantes e otros muchos otros secretos de la Natura. Digo de lo que estaba ya experimentado o sabido por los naturales destas nuestras isla; e todo lo que agora se puede decir, es poco e no bien entendido, porque esta generación es tan avara deso poco que sabe, que por ningún interese ni bien que se les haga quieren manifestar cosa déstas, en especial de las que podrían aprovechar a los cristianos, si son medecinales, porque esta manera de sciencia es parte de su señorío. ${ }^{18}$

Aunque imperfecta, el intento de restitución de la especiería vislumbra tímidamente una emergente dimensión culinaria. La identificación de especias, como posibles nuevos sabores y condimentos del territorio indiano, constituyen un medio por el cual lo desconocido (el mare magno) se hace accesible a Fernández de Oviedo y sus lectores. Pero también su enunciación como especias, es decir, como condimentos, medicinas y fármacos propios del Nuevo Mundo, comienzan a dar forma a una nueva

17 Ibidem, lib. X, cap. III, 12-13.

18 Ibidem, lib. X, cap. VIII, 20. La postura de Fernández de Oviedo frente a la población del Nuevo Mundo es un tema recurrente en la historiografía sobre el cronista, con planteamientos que frecuentemente han oscilado entre el panegírico y el libelo. Véase, entre otros: Peña y Cámara (1957), Vázquez (1957), Bolaños (1990), Coello de la Rosa (2001), Aram (2008). 
clasificación que se despliega paralelamente a la topología descriptiva de la naturaleza indiana. El vínculo no es arbitrario. Para el siglo XVI, mientras los planteamientos de Hipócrates y Galeno siguen vigentes, la farmacopea no es mucho más que una dietética. Alimento y fármaco, especia y medicina se encuentran íntimamente ligados. Seña de ello es que la noción de receta se emplea tanto para la preparación de platos como para la prescripción farmacológica. ${ }^{19}$

\section{La búsqueda del sabor vegetal}

En este sentido, no basta con identificar qué elementos del Nuevo Mundo son comestibles. Para los dietistas del siglo XVI, el buen gusto y la buena digestión están íntimamente asociados: no solo porque se digiere mejor lo que se come con gusto, sino porque se cree que si un alimento gusta es señal de que conviene al temperamento de quien lo ingiere. ${ }^{20}$

Es así como Fernández de Oviedo examina los frutales del Nuevo Mundo desde su memoria gustativa. Dar cuenta de los sabores de la naturaleza del Nuevo Mundo es por tanto una operación doble: por una parte, implica también dar cuenta de su utilidad en tanto imaginario de especiería; pero por la otra, remite a organizar la naturaleza comestible, permitiendo establecer una pauta para la instauración de una dieta colonial. ${ }^{21}$

Nuevamente, es la semejanza - la mimesis con lo conocido- la que opera en un primer momento para identificar los productos del Nuevo Mundo. Fernández de Oviedo encuentra recurrentemente «higos», «manzanas», «peras» y «avellanas» que se asimilan a sus versiones europeas. Parecido formal, pero también gustativo (como la acana que sabe a queso), de texturas y sensaciones que sirven para aproximarse imperfectamente a lo desconocido. Así encara la guanábana, cuya fruta es

hermosa e grande, como melones en la grandeza [... por] afuera tienen escamas como piñas, más lisas que aquellas señales e no levantadas como las de las piñas [... y] el cuero o corteza es delgado, como el de una pera, o poco más, e la fructa e manjar de dentro es como natas, o manjar blanco al parecer, porque hace alguna correa. Esta comida o manjar se deshace luego en la boca, como agua, con un dulzor bueno. Y entre

19 Contreras y García, 2005, 70-71.

20 Flandrin, 2011, 634.

21 El primer punto ha sido brevemente tratado en Baraibar, 2014, 11-12. Lo segundo ha sido apuntado de forma genérica por Carrillo, 2004, 148. 
aquellas carnosidades, hay asaz pepitas grandes, como las de la calabaza, pero más grosezuelas, de color leonadas oscuras. Son, como he dicho, altos e grandes e hermosos árboles, e muy fresca e verdes las hojas, e cuasi a la hechura de la hoja de la lima.22

Melones, piñas, peras, manjar, calabaza y lima son necesarios para transmitir al lector las características de las frutas que se encuentran en el Nuevo Mundo. Para evocar lo desconocido, Fernández de Oviedo debe echar mano a una tradición compartida de prácticas alimentarias, saberes y sabores. Como plantea Alexadre Coello de la Rosa, la descripción de Fernández de Oviedo aún se encuentra anclada en el lenguaje y la mentalidad europea. ${ }^{23}$ El caso límite de la evocación de lo desconocido es, tal vez, la descripción que realiza de la piña, donde pone en práctica cuatro sentidos, para terminar asegurando que es indescriptible: ${ }^{24}$

Esta es una de las más hermosas fructas que yo he visto en todo lo que el mundo he andado. [...] Ni pienso que en el mundo la hay que se le iguale en estas cosas juntas que agora diré. Las cuales son: hermosura a la vista, suavidad de olor, gusto de excelente sabor. [...] Mirando el hombre la hermosura désta, goza de ver la compusición e adornamiento con que la Natura la pintó e hizo tan agradable a la vista para recreación de tal sentido. Oliéndola, goza el otro sentido de un olor mixto con membrillos e duraznos o melocotones, y muy finos melones, y demás excelencias que todas estas fructas juntas y separadas, sin alguna pesadumbre; y no solamente la mesa en que se pone, mas, mucha parte de la casa en que está, siendo madura y de perfecta sazón, huele muy bien y conforta este sentido del oler maravillosa e aventajadamente sobre todas las otras fructas. Gustarla es una cosa tan apetitosa e suave, que faltan palabras, en este caso, para dar al propio loor en esto; porque ninguna de las otras fructas que he nombrado, no se puede con muchos quilates comparar a ésta. Palparla, no es, a la verdad, tan blanda e doméstica, porque ella misma parece que quiere ser tomada con acatamiento de alguna toalla o pañizuelo; pero puesta en la mano, ninguna otra da tal contentamiento. [...] No pueden la pintura de mi pluma y palabra dar tan particular razón ni tan al propio el blasón desta fructa, que satisfaga tan total y bastantemente que se pueda particularizar el caso sin el pincel o debujo, y aun con esto, sería menester las colores, para que más conforme (si no en todo, en parte), se diese mejor a entender que yo lo hago e digo, porque en alguna manera la vista del letor pudiese más participar desta verdad. ${ }^{25}$

22 Fernández de Oviedo, lib. VIII, cap. XVII, 1992, 1, 258.

23 Coello de la Rosa, 2002a, 82.

24 Para Coello de la Rosa, 2002b, lo indescriptible de la naturaleza indiana en Fernández de Oviedo se corresponde con lo inconmensurable de la divinidad cristiana y su obra. Ambos, se presentan como un exceso de significado que maravilla mientras desafía la capacidad para percibir la realidad.

25 Fernández de Oviedo, lib. VIII, cap. XIV, 1992, 1. Sobre la idea de lo indescriptible de la naturaleza indiana, Jesús Carrillo plantea que la densidad sensorial de las descripciones de Fernández de Oviedo son el producto de una insuficiencia en el lenguaje para nombrar, Carrillo, 2003, 491. 
Utilizar el imaginario de los alimentos del Viejo Mundo no solo implica disminuir la brecha de referencia estética que permita vincular los nuevos sabores al sistema alimentario europea, sino también, es una forma de inscribir lo nuevo en una tradición simbólica asociado a una dietética particular. Un caso paradigmático de esta actitud es, tal vez, la descripción que Fernández de Oviedo realiza del aguacate, al cual identifica con una especie de pera salvaje. Dice así,

la fructa que llevan son peras en el talle y en la color, e no más, porque el cuero es tan gordo como de un borceguí de cordobán, e la carnosidad de dentro no es más gruesa que de una pluma de escribir de un ansarón, o cuando más como la de un cisne; e el cuesco es tan grande, que ocupa todo lo demás; y no cuesco, sino pepita, cubierta de una telica delgada que proveyó Natura, porque lo que se come desta fructa no tocase la pepita que es amarguísima. ${ }^{26}$

Convencido de que la forma determina la identidad del fruto, Fernández de Oviedo llega a comparar el sabor de peras y aguacates, como si se tratasen efectivamente del mismo fruto:

desque están maduras, fácilmente se deja cortar aquella corteza que tiene, y se despide por sí misma la pepita de en medio con su telilla, e la corteza asimismo, e lo que queda parece manteca e es gentil manjar, e yo le tengo por mejor que las peras de Castilla. ${ }^{27}$

Sin embargo, la exacerbación del gesto se alcanza cuando expone con aparente ingenuidad la versión indiana de una conocida receta proveniente del repertorio culinario galénico, a saber, las peras con queso: «con queso sabe muy bien estas peras, y cuando están sazonadas para las comer, piérdense, si las dilatan e dejan pasar aquella sazón, porque se acedan y pudren, e no valen nada si con tiempo no las conceden al gusto».28

Aunque presente en múltiples refranes populares del Viejo Mundo, la asociación de las peras con el queso responde también a una tradición médica alimentaria. Considerado alimento popular, hacia fines del medievo la nobleza sospecha de la calidad digestiva del queso, que progresivamente comenzaba a apreciar. Para conjurar lo nocivo de este, el médico italiano Castor Durante de Gualdo recomienda en 1565 comérsele con peras a fin de contrarrestar sus efectos. ${ }^{29}$ De algún modo, Fernández de Oviedo

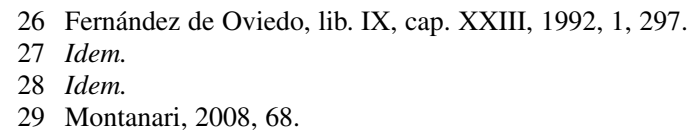


hace eco de esta tradición, y de paso reinscribe los frutos del Nuevo Mundo en un imaginario dietético que, junto a la noción de especias, busca poner en valor la naturaleza de Indias.

\section{Organizar los animales}

Sin duda es en la fauna indiana donde la emergencia de una trama clasificatoria paralela - frente a una historia natural, otra culinaria - puede apreciarse con más claridad. Como se propone para la totalidad de la naturaleza indiana, la intención original de Fernández de Oviedo es complementar el saber de Plinio con los animales del Nuevo Mundo. Para ello, y siguiendo el principio de semejanza, replica las cuatro categorías de Plinio para el mundo animal: los terrestres, los acuáticos, las aves y los insectos.

No obstante, identificar los animales del Nuevo Mundo no va a ser tarea fácil, la fauna que cree reconocer en la tradición pliniana va a estar sujeta a un método de contrastes entre las opiniones de los clásicos, los testimonios de las crónicas indianas y su propia experiencia en el Nuevo Mundo. Como ocurre en el caso de los cocodrilos del río de la Plata, donde tras la descripción de ciertos animales que comen los indígenas cita la caracterización de Isidoro de los cocodrilos del Nilo, para concluir:

por manera que aquestos lagartos del río de la Plata serían cocatrices cuanto al mover
la mandibula o mejilla alta, y no cuanto a la color, pues Isidoro dice que del croceo
color, se llama cocodrilo: luego estos no lo serían, pues no son amarillos ni tan gran-
des como los del Nilo. Lo que yo pienso es que lo son, aunque no sean de aquella
color, porque ésta no es tan bastante señal para dejarlo de creer, como es, para haber-
lo por cierto, mover la mejilla alta..$^{30}$

En principio, las cuatro categorías que utiliza para clasificar la fauna no le son problemáticas y, en apariencia, son excluyentes. Sin embargo, no hay ordenamiento que no presente anomalías y para Fernández de Oviedo esta se encuentra en la iguana. La iguana en la obra indiana de Fernández de Oviedo representa el paradigma del problema de la clasificación. En el Sumario realiza una primera descripción del animal. Cuando habla de los mantenimientos indígenas de La Española, plantea que

30 Fernández de Oviedo, lib. XII, cap. VII, 1992, 2, 360. 
comían asimismo una manera de sierpes que a la vista son muy fieras y espantables, pero no hacen mal, ni está averiguado si son animal o pescado, porque ellas andan en el agua y en los árboles y por tierra, y tienen cuatro pies, y son mayores que conejos, $\mathrm{y}$ tienen cola como lagarto, $\mathrm{y}$ la piel toda pintada, y de aquella manera de pellejo, aunque diverso y apartado en la pintura, y por el cerro o espinazo unas espinas levantadas, y agudos dientes y colmillos, y un papo muy largo y ancho, que le cuelga desde la barba al pecho, de la misma tez o suerte del otro cuero y callada, que ni gime ni grita ni suena, y está se atada a un pie de un arca, o donde quiera que la aten, sin hacer mal alguno ni ruido, diez, y quince, y veinte días, sin comer ni beber cosa alguna. ${ }^{31}$

La iguana emerge como un animal indeterminado, sin embargo, en principio esto no se debe a que sea inclasificable, sino al escaso conocimiento que se posee de nuevo animal. Asimismo, para el Sumario la clasificación no es un problema ya que la fauna indiana aparece bajo una sola categoría, a saber, la de animales.

Es, no obstante, en la primera edición de la primera parte de su Historia (1535) que Fernández de Oviedo introduce la clasificación de la fauna indina, y decide ubicar a la iguana junto a los peces. En principio esto no debiera ser problemático. Desprovisto de criterios taxonómicos modernos la decisión de Fernández de Oviedo puede parecer tan arbitraria como su opuesta. El problema deviene en la segunda edición, cuando Fernández de Oviedo ubica a la iguana dentro de los animales terrestres del Nuevo Mundo. ¿Qué ocurre entonces? Aunque no contamos con acceso al texto de 1535, en la segunda edición de la primera parte de la Historia Fernández de Oviedo explica las razones que lo llevaron a incluir a la iguana en la categoría de los peces: a su modo de ver, la iguana sería un animal que vive tanto en los ríos como en los árboles, y, lo que es más importante, no existe un consenso si es carne o pescado. ${ }^{32}$ Pero la inclusión en los animales terrestres en la segunda edición tampoco soluciona el problema, sino que opera como una declaración sobre la carencia de clasificación del animal.

Agora me pareció ponerle en éste que tracta de los animales terrestres, non obstante que, segund la opinión de muchos, que entrambos libros se puede aplicar, porque muchos hombres hay que no se saben determinar si este animal es carne o pescado, e como cosa neutral, la atribuyen al uno y al otro género, así de los animales de la

31 Fernández de Oviedo, 1996, 99-100.

32 Jesús Carrillo ve en la reclasificación de la iguana que hace Fernández de Oviedo la expresión ejemplar de la confrontación de la experiencia personal con la autoridad, representada por Pedro Mártir; es decir, el hecho grafica la posibilidad de ubicar al Nuevo Mundo en una dimensión autónoma frente al mundo clásico (incluido Plinio). Carrillo, 2003, 497. 
tierra como de los del agua, porque así se aplica al un elemento como al otro, y en cada uno de ellos se ejercita y continúa la vida. ${ }^{33}$

La aparente neutralidad de la iguana, su dificultad clasificatoria, responde a un problema teológico antes que epistemológico. Determinar si es carne o pescado, más que un tema filogenético alude a discusiones religiosas. Como el mismo Oviedo indica, independiente de dónde se clasifique «usan de él en estas partes, comiendo este animal en los días que no son de carne, así como viernes e sábado, e la cuaresma, e otros días prohibidos por la Iglesia. Más de mi opinión e parescer, yo le habría por carne. Lo cual no digo para que ninguno deje de seguir su voluntad, y principalmente la del prelado y lo que la Iglesia ordenare». ${ }^{34}$

En cierto sentido, el problema de la iguana ${ }^{35}$ demuestra cierta limitación del modelo de Plinio para dar cuenta de los animales del Nuevo Mundo. Aunque la clasificación clásica posee utilidad para ordenar una serie de animales desconocidos en el Viejo Mundo, el dilema de la iguana abre en Fernández de Oviedo una segunda línea de clasificación de la fauna indiana. Como hemos venido sosteniendo, es fácil constatar en la lectura de la obra indiana de Fernández de Oviedo la especial preocupación por dar cuenta de las posibilidades culinarias de la naturaleza de Nuevo Mundo. En el caso de la iguana, su problema clasificatorio no es taxonómico, sino alimentario: esta no es más pez por nadar o no hacerlo, sino por la calidad de su carne. Por ello, tras la descripción que Fernández de Oviedo realiza en el Sumario, concluye afirmando que «es muy mejor de comer que de ver» ${ }^{36}$ pero es en la Historia donde el potencial culinario de la iguana es profundizado con mayor ímpetu. Luego de describir profusamente los métodos de cocción de la iguana, Fernández de Oviedo se dedica a explicar cómo deben ser cocinados los huevos del animal:

33 Fernández de Oviedo, lib. XII, cap. VII, 1992, 2, 32.

34 Idem. Sobre este punto Jeremy Paden plantea que la clasificación final que Fernández de Oviedo realiza de la iguana responde, en última instancia, a una memoria cultural, que inserta el animal en el imaginario cristiano de semana santa. Paden, 2007.

35 Si la iguana es para Fernández de Oviedo un animal terrestre cuya carne puede confundirse con un pez, el manatí ocupa una posición inversamente simétrica, pues siendo un animal marino su carne se asemeja a la de los animales terrestres: «creo que es uno de los buenos pescados del mundo y el que más parece carne; y en tanta manera parece vaca, viéndose cortado, de quien no le obiere visto entero o no supiere, mirando una pieza dél, no sabrá determinar si es vaca o ternera». Fernández de Oviedo, lib. XII, cap. IX, 1992, 2, 64.

36 Fernández de Oviedo, 1996, 100. 
Y como experimentado, quiero avisar a quien esto leyese en estas partes (si indios faltaren, como faltan), de la manera e arte que han de tener para guisar los huevos de la iuana, porque hallarán por verdad, que queriendo hacer una tortilla de los huevos, o freírlos como los que dicen estrellados, no se podrá hacer con aceite ni manteca, porque nunca se cuajarán; mas, echando agua en lugar de aceite, se cuajan e guisan. Esto es cosa probada e cierta, e otro indicio para porfiar a sabiendas los que menos entienden, que éste es pescado, e tan amigo del agua, que se conforma más con ella que con los materiales de la tierra. Pero esto es falso, o no decir nada, pues que todos los pescados, o los más dellos se guisan e fríen con aceite. ${ }^{37}$

Desde esta óptica, la distribución de los animales indianos se transforma en una suerte de compendio gastronómico, en el cual tras cada descripción física casi necesariamente se acompaña de un comentario culinario sobre el sabor de la carne o métodos de preparación. Incluso cuando Fernández de Oviedo no ha probado determinado animal, remite a algún testimonio para dar cuenta de su sabor. Como la hutía en La Española, que se encuentra en extinción por el consumo de los conquistadores pero que «segund testifican muchos que los vieron e comieron [...] los loan por buen manjar». ${ }^{38}$ Así mismo, entre los animales de esa isla se encuentra el quemí, de buen sabor, aunque en la opinión de Fernández de Oviedo sobrevalorado pues los primeros colonos pasaron mucha hambre; el mohuy, altamente estimado por los caciques y del cual «los hombres que lo vieron y comieron [...] loan esta carne por mejor que todas»; ${ }^{39}$ el corí, una especie de conejo pero cuya carne es menos seca que este animal; los perros gozques, ya extintos por el sobre consumo de los primeros colonizadores, pero «todos los españoles que los han probado, loan este manjar e dicen que les parece no menos bien que cabritos»;40 y la ya referida iguana. De los animales de La Española, solo los ratones y las culebras, ambos considerados perniciosos, no están acompañados de un comentario culinario.

El mismo gesto se replica cuando Fernández de Oviedo revisa la fauna de Tierra Firme. Aquí encuentra al beori, del que alaba su carne y entrega la receta para comer sus patas: «los pies deste animal son muy buen

37 Fernández de Oviedo, lib. XII, cap. VII, 1992, 2, 33.

38 Ibidem, cap. I, 29.

39 Ibidem, cap. III.

40 Ibidem, cap. V, 30. En su búsqueda por la rigurosidad de sus juicios culinarios, Fernández de Oviedo afirma haber probado en Nicaragua un perro similar, llamado xulo, tan preciado por los indígenas que la cabeza es privativa de los calachuni o teite, es decir, los principales. «Y a la verdad, de aquél que yo comí, fueron dos o tres bocados, en no pensando que era perro. E llegué donde ciertos amigos comían de uno muy gordo e muy bien asado e untado o lardado con ajos, e no me supo mal». Idem. 
manjar, salvo que es menester que se cuezan veinte horas o más (quiero decir que estén muy cocido), porque tardan en cocer. Mas estando tales, es manjar para darle a cualquiera que huelgue de comer una cosa de muy buen gusto o digestión»; ${ }^{41}$ al ciervo, que en Nicaragua se conoce como mazat dice que «es de muy buena carne, y en todo tiempo del año, en especial en esta provincia de Nicaragua y en León de Nagrando»; ${ }^{42}$ a los conejos y liebres, los que explica cómo los indígenas los conservan en sal, «así en cecina para cuando les falta la carne fresca» ${ }^{43}$; y los encubertados (armadillos), de los que afirma son excelente manjar:

quitándoles aquella concha, están muy gordos, e cuasi lo más dellos cubiertos de grasa o manteca sobre la carne. E porque toman mucho la sal, e sin ella son muy dulces, no los comen sino salados de un día antes, porque no echándoles sal, son tan gordos que empalagan o dan fastío; pero es buena carne. Yo los he comido algunas veces, e son mejores que cabritos en el sabor, e es manjar sano. ${ }^{44}$

La lista prosigue con ardas, ovejas de Nueva Castilla (auquénidos) - que a su parecer tienen una de las mejores carnes del mundo- y vacas septentrionales (bisonte); para luego continuar con los peces («el manjar más ordinario de los indios e a que ellos tienen grande afición. [...] Y a mi creer, estos pescados de acá son más sanos que los de España, porque son de menos flema, pero no de tan buen sabor»), ${ }^{45}$ las aves, e insectos (principalmente chapulines) indianos.

Pues bien, esta segunda trama clasificatoria (la culinaria) no tendría sentido si solo existiese una categoría constituida por los animales comestibles. Junto a los animales «buenos para comer» existen también aquellos que no lo son. Como el oso hormiguero, del cual tras explicar su estrategia alimenticia, afirma que

la carne deste animal es sucia e de mal sabor; pero como las desventuras e necesidades de los españoles en aquellas partes, en los principios, fueron muchas e muy extremadas, no se han dejado de probar a comer; pero hace aborrecido tan pronto tal manjar, como se probó por algunos cristianos. ${ }^{46}$

41 Ibidem, lib. XII, cap. XI, 43.

42 Ibidem, cap. XIX, 45.

43 Ibidem, cap. XXII, 47.

44 Ibidem, cap. XXIII, 48

45 Ibidem, lib. XIII, cap. I, 57.

46 Ibidem, lib. XII, cap. XXI, 47. 
$\mathrm{O}$ un animal llamado aire que «es muy duro de comer [...] y es que después de cocidos, aunque mucho más los cuezan, no están por eso más tiernos de comer, ni tampoco porque mucho los asen». ${ }^{47}$

Aunque la oposición conforma ya un incipiente sistema clasificatorio, Fernández de Oviedo establecerá una tercera categoría alimentaria. Junto a los animales buenos y malos para comer, emergerá aquella conformada por los animales que «se alimentan» de los humanos. Algunos lo hacen en un sentido literal: como el tigre o pantera, que los indios llaman ochi, de los cuales «hay muchos dellos en Tierra Firme, e comen a muchos indios, e son muy dañosos» ${ }^{48} \mathrm{o}$ los lobos de la Tierra Firme que «son bermejos e malos, e comen algunos indios». ${ }^{49}$ Pero también indirectamente, matando o alimentándose del sustento de la población indiana. Como los puercos monteces que matan perros, las churchas que degüellan gallinas, o la broma que acaba con la madera de las construcciones.

Aunque la clasificación es incompleta ${ }^{50}$ da cuenta de la preocupación de Fernández de Oviedo por hacer de las Indias un lugar sustentable y útil para la colonización. Junto con plantear una solución al problema de las categorías cognitivas, la inscripción de la naturaleza en el orden alimentario permite transformar parte de su obra indiana en una guía práctica para la colonización; al mismo tiempo que, como afirma Coello de la Rosa, la naturaleza se establece un principio de organización en sí mismo ${ }^{51}$. Así, restituir el imaginario de especiería no solo significa reinscribir un valor extractivo en el Nuevo Mundo, sino también hacer de él un espacio ordenado y, por lo tanto, habitable. Esto explica en parte que, junto a la clasificación alimentaria de la naturaleza, Fernández de Oviedo se preocupe por indicar no solo las propiedades alimentarias de la naturaleza salvaje, sino también de aquella culturalmente domesticada por la población del Nuevo Mundo.

47 Ibidem, cap. XXXIV, 54.

48 Ibidem, lib. XII, cap. X, 39.

49 Ibidem, lib. XII, cap. XVI.

50 Si bien este ordenamiento abarca la gran mayoría de los animales a los que Fernández de Oviedo se refiere en su obra indiana, existen unos cuantos que quedan desclasificados, como el perico ligero (el perezoso), del cual no se sabe con certeza de que se alimenta y, aunque no ponzoñoso, tampoco es destinado a la alimentación, por lo que concluye: no «he visto hasta agora animal tan feo ni que parezca ser tan inútil que aqueste». Ibidem, lib. XII, cap. XXIV, 42.

51 Coello de la Rosa, 2002a, 73. 


\section{Los panes indianos y la aproximación culinaria}

La trama alimentaria como seña clasificatoria de la naturaleza abre, en Fernández de Oviedo, una nueva dimensión para comprender las Indias. Junto a esta, la perspectiva culinaria permite también dar cuenta de la población del Nuevo Mundo. Desde la descripción que realiza en el Sumario de los indígenas de La Española, el mantenimiento — «lo que comen»- se transforma en un elemento constitutivo de la población. Como si hiciera eco de la mitología prehispánica que posiblemente desconocía, Fernández de Oviedo le asigna una importancia capital a los panes indianos, hechos de maíz y cazabe (yuca). Lo más probable es que este gesto, antes que una apertura a la simbología del otro, estuviese marcado por su propia tradición. Como afirma Sophie Coe:

para los aztecas, los mayas, los incas y los europeos del siglo XVI, el pan era la fuente de todos los carbohidratos de mayor importancia, su falta significaba hambruna, y su presencia, incluso por sí solo, indicaba que ya se estaba alimentado y satisfecho. ${ }^{52}$

En muchos sentidos, el conocimiento sobre la alimentación de los indígenas del Nuevo Mundo no es más que el suplemento del problema clasificatorio de la naturaleza. Desde la imagen del mare magno, este remite a un saber oculto, sujeto a ser descifrado. Como hemos visto, la clave de ello se encuentra en la alimentación: como especia, como comida, como medicina. Dar cuenta de la alimentación de los habitantes del Nuevo Mundo es, en este sentido, dar cuenta también de un desciframiento previo de la naturaleza, de un saber que ha sido culturalmente codificado en ciclos productivos, distributivos, elaboraciones y consumo. Y en esta línea, describir estos ciclos se corresponde al gesto de apropiación que tiene el ordenar y clasificar la naturaleza del Nuevo Mundo. Es así como Fernández de Oviedo explica en la Historia las técnicas y saberes agrícolas en torno al maíz ${ }^{53}$ y la yuca, ${ }^{54}$ con el fin de que la población colonizadora aprenda a cultivarlos. Pero es sin duda en la dimensión culinaria donde la importancia del saber en torno estos alimentos es fundamental. Si los frutos y los animales de la naturaleza daban pie un incipiente recetario indiano, los alimentos domesticados se encuentran culturalmente recubiertos de una serie

52 Coe, 2004, 26.

53 Fernández de Oviedo, lib. VII, cap. I, 1992, 1, 226-227.

54 Ibidem, cap. II, 230. 
de técnicas, procedimientos, recetas y combinaciones que Fernández de Oviedo registra acuciosamente. Para el caso del maíz apunta lo siguiente:

En la isla Española y en otras, comíanlo en grano tostado o, estando tierno, sin tostar, cuasi seyendo leche; e cuando es así tierno, llámalo ector, queriendo cuajar o recién cuajado. [...] En Tierra Firme tienen los indios otro uso [...] y es de aquesta manera. Las indias, en especial, lo muelen en una piedra de dos palmos o más o menos, de longitud, e de uno y medio o dos de latitud, cóncava, con otra redonda o rolliza y luenga que en las manos traen, a fuerza de brazos (como suelen los pintores moler colores para su oficio), echando agua e dejando pasar algún intervalo, poco a poco, no cesado el moler. E así se hace una manera de pasta o masa, de la cual toman un poco e hacen un bollo de un jeme, e grueso como dos o tres dedos. Y envuelta en una hoja de la misma caña del maíz, u otra semejante, y cuecenlo. [...] Y si no lo quieren cocer, asan esos bollos en las brasas, al resplandor, cerca dellas, y enduréscese el bollo, y tornase como pan blanco, e hace su corteza por de suso, y de dentro hace miga algo más tierna que la corteza, e quítanle la hoja en que lo envolvieron para lo cocer o asar, e cómenlo algo caliente, y de no del todo frío; porque si se enfría no tiene tan buen sabor ni es tan bueno de mascar. [...También] usan unas tortas grandes, delgadas e blancas, el arte de las cuales procedió de la Nueva España, así en México como en otras provincias della. [...] Este tal pan se llama tascalpachon, y es muy buen pan sabroso. Hacense otras tortas de la misma masa del maíz, escogiendo para ello el grano más blanco, e despican los granos, antes que los muelan, quitándoles una dureza u raspa que tienen en el pezón con que estovieron pegados en la espigao mazorca; e así sale mejor e más tierno el pan, e no se topan entre los dientes aquellas durezas que se topan cuando los bollos e tortillas son de maíz que no fue despicado. ${ }^{55}$

Fernández de Oviedo continúa con su descripción de los usos alimentarios del maíz hablando de las bebidas: maíz fermentado que se ingiere en Nueva España y la harina de maíz tostada (pinole) que consumen - mezclada con agua - los indígenas durante los viajes. La descripción culinaria de las elaboraciones indígenas cumple distintos fines: representa la dimensión cultural que envuelve la naturaleza indiana, es decir, da cuenta de cómo la población local aprovecha sus recursos; pero también se presenta como un modo de apropiación del saber indígena para los fines de la colonización. Así, tras la descripción culinaria del maíz Oviedo introduce un comentario que da cuenta de esta apropiación por parte de los españoles:

los cristianos han dado mucha mejoría a este pan, cociéndolo en horno a la manera de España, e es más sabroso e más lindo en la vista, así cocido, en roscas o tortas. E hácese asaz buen biscocho dello, para navegar con ello no muy largo tiempo. ${ }^{56}$

55 Ibidem, lib. VII, cap. I, 228-229.

56 Idem. 
A pesar de que es un lugar común la referencia a que la población europea tardó en asimilar los alimentos indianos, ${ }^{57}$ para quienes vivían en el Nuevo Mundo es probable que sus márgenes de elección fueran bastante estrechos. En este sentido, los comentarios culinarios de Fernández de Oviedo, así como la descripción del sistema productivo, implican un intento larval de sistematizar un saber necesario para la relación colonial.

Ahora bien, no solo de maíz (y de yuca) ${ }^{58}$ se compone la culinaria del Nuevo Mundo que capta la atención de Fernández de Oviedo. Ya hemos visto cómo la descripción de la flora y la fauna está atravesada por una preocupación alimentaria, la cual a momentos adquiere un verdadero cariz gastronómico. Si a Fernández de Oviedo se le ha atribuido ser precursor de un naturalismo europeo en Indias, ${ }^{59}$ así como el creador de uno de los primeros bestiarios indianos,${ }^{60}$ no es osado afirmar que ciertos pasajes de su Historia componen uno de los primeros recetarios mestizos del Nuevo Mundo.

Aunque no hay certeza a qué planta remiten los ajes, ${ }^{61}$ parientes — para Fernández de Oviedo— de las batatas, afirma que:

cocidos son muy buenos, e asados tienen algo mejor sabor, y de la una o de la otra manera tienen sabor de castañas muy buenas; porque, como no la comen por principal y ordinario manjar, sino de cuando en cuando, sabe mejor. Asados e con vino, son buenos de noche, sobre mesa; e en la olla son buenos. Las mujeres de Castilla hacen diversos potajes e aun fructa de sartén, e tal que, aunque fuese de Indias, se sabría por buena. Son los ajes de buena digestión, aunque algo ventosos. ${ }^{62}$

Fernández de Oviedo probablemente no solo replica el saber indígena de la cocción de los ajes, sino que además — como en el caso que ya vimos del aguacate con queso- introduce elementos del Viejo Mundo (el vino) que, a su gusto, mejoran la preparación. Asimismo, destaca el hecho que

57 Por ejemplo, para el caso del maíz ver Brandes, 2011.

58 Oviedo dedica un análisis similar a la yuca y sus múltiples versiones y procesamientos. Ver Fernández de Oviedo, lib. VII, cap. II, 1992, 1, 230-232

59 Regueiro, 1983.

60 Rodilla, 2007.

61 Siguiendo una línea que viene de Colón, Oviedo identifica los ajes con el ñame, aunque por su descripción es más probable que sea una papa: «hay una planta se llama ajes, los cuales quieren parecer algo, en la vista, a los nabos de España, en especial los que tienen la corteza o tez de encima; porque estos ajes haylos blancos, y colorados que tiran a morado, y otros como leonado; pero todos son blancos de dentro, por la mayor parte, y algunos amarillos, y muy mayores que nabos, comúnmente. Críanse debajo de tierra, e hacen encima de tierra una rama tendida a manera de correhula, pero más gruesa; la cual con sus hojas e ramas, cubre toda la superficie de la tierra do están sembrados los ajes». Fernández de Oviedo, lib. VII, cap. III, 1992, 1, 233.

62 Ibidem, 234. 
las mujeres castellanas se han apropiado del alimento, incluyéndolo en su dieta indiana. Algo similar ocurre con la batata, parecida a los anteriores, solo que es

más delicada fructa o manjar, y el cuero o corteza más delgada, y el sabor aventajado y de mejor digistión. Una batata curada no es inferior, en gusto, a gentiles mazapanes. [...] E se comen cocidas o asadas, y en potages e conservas, e de cualquier forma son buenas fructas, e se pueden presentar a la Cesarea Magestad por muy preciado manjar. $^{63}$

Fernández de Oviedo vuelve a evocar sabores foráneos, y aunque es probable que el curado también fuese una técnica indiana, indudablemente alude a preparaciones europeas. Si el mestizaje culinario se produce por la incorporación de ingredientes y técnicas de cocina, también se encuentra presente por oposición. De la piña, que ya hemos mencionado, dice que una vez mondada «hácenla tajadas redondas, o chullas, o como quiere el trinchante, porque en cada parte, al luengo o a través, tiene pelo e gentil corte» $;{ }^{64} \sin$ embargo esta fruta, que Fernández de Oviedo considera casi perfecta, posee un único problema,

por el cual no agrada complidamente a todos los gustos; y es que el vino, aunque sea el mejor del mundo, no sabe bien bebido tras la piña, e si así supiera como sabe con las peras asaderas, u otras cosas que con el beber tienen aprendido los que son del vino amigos, fuera única a su parecer de los tales. ${ }^{65}$

Otro tanto ocurre con el plátano que, como apunta Coe, era

una planta domesticada en el Viejo Mundo, pero que fue una de las que se extendieron con mayor rapidez por la zona tropical del Nuevo Mundo; tanto que incluso algunos de los primeros cronistas se equivocaron, considerándola una planta nativa de América. ${ }^{66}$

Aunque Fernández de Oviedo no entra dentro de este grupo de cronistas, pues sabe que no «los había en Indias, e fueron traídos a ellas», ${ }^{67}$ va a dedicarles un importante espacio a la culinaria que en Indias se practica con ellos:

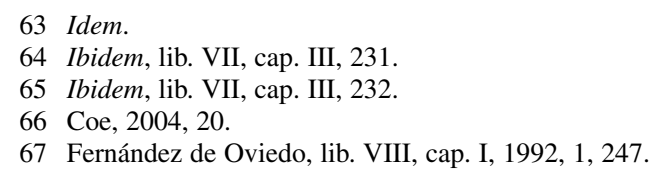


Hase cortar el racimo desta fructa, así como un fructo de los que están en racimo se comienza a hacer amarillo, e después, el racimo entero cuélganlo en casa, a allí se madura toda la fructa dél (o todos los plátanos que en el racimo hay). Esta es una buena fructa, e cuando se curan estos plátanos abiertos al sol, hendiéndolos con un cuchillo en dos mitades al luengo, e dándoles sendas cuchilladas, o cada dos o cada mitad, cortando la fructa al luengo hasta la cascara, e no rompiendo la cascara o cuero, hácese el sabor, cuando están curados, muy semejante a los higos pasos, y aun mejores. En el horno asados, sobre una reja u otra cosa semejante, son muy buena e sabrosa fructa, e parece un género sobre sí, como lo es, de una conserva melosa e de muy buen cordial e suave gusto. Asimismo, cociéndolos en la olla con la carne, es un buen manjar; pero no ha de estar el plátano mucho duro para lo cocer con la carne, ni muy maduro, ni se ha de echar sino cuando esté la carne cuasi cocida, e desollado; porque en uno o dos hervores, en poco espacio de tiempo, se cuece el plátano. Comidos crudos, después que maduran, es muy gentil fructa, y no es menester comer con ella pan ni otra cosa, y es de excelente sabor, e sana e de gentil digestión: que nunca he oído decir que hiciese mal a ninguno. Llevandolos por el mar, duran algunos días, e hanse de coger para esto algo verdes; e lo que turan sin se podrir o dañar (que es doce o quince días), saben mejor en la mar que en la tierra (como hacen las cosas deseadas, donde menos se pueden haber). ${ }^{68}$

\section{Las nuevas especias indianas}

Llegados a este punto no cabe duda que la clave culinaria puede ser pensada pertinentemente como una trama paralela a la hora de evaluar la aproximación y clasificación que Fernández de Oviedo hace de la naturaleza del Nuevo Mundo. Sin embargo, aunque de la batata diga que puede ser servida en la mesa del rey, las preparaciones que anota de los productos indianos tienen una función bastante más local. De algún modo, y como gran parte de la obra, los comentarios culinarios poseen una función pedagógica sobre la población colonizadora, ${ }^{69}$ Enseñan qué cultivan, cómo lo preparan y qué come la población indígena, pero también entrega una guía gustativa del Nuevo Mundo a los españoles que participan de la colonización. Así, la utilidad de la flora y fauna indiana está dirigida, en su mayor parte, a sustentar el ordenamiento colonial.

Sin embargo, como hemos venido planteando, junto a la dimensión culinaria Fernández de Oviedo introduce algunos comentarios que intentan restituir el imaginario de especiería en Indias: diversas tinturas, plantas

68 Ibidem, lib. VIII, cap. I, 248.

69 Sobre este punto ver Bolaños, 1991. 
medicinales, la canela del Perú conforman este repertorio que soterradamente otorga una utilidad comercial al Nuevo Mundo. Corresponde también incluir al ají, que desde una tradición que se inicia con Colón, es visto por Oviedo como la pimienta indígena:

es caliente e da muy buen gusto e apetito con los otros manjares, así al pescado como a la carne, e es la pimienta de los indios, y de que mucho caso hacen, aunque hay abundancia de ají, porque en todas las labranzas e huertos lo ponen e crían con mucha diligencia e atención, porque continuamente lo comen con el pescado y con los demás manjares..$^{70}$

Pero es sin duda su reflexión en torno al azúcar la que intenta concretar de manera más efectiva este imaginario. En el capítulo VIII del libro IV, dedicado a La Española, Fernández de Oviedo se extiende largamente en la emergencia y proliferación que han tenido los trapiches de azúcar en la isla. Leído desde el presente, resulta extraña la prioridad que le concede a la caña sobre otros cultivos. Sin embargo, aunque en la actualidad pueda parecernos un producto relativamente barato, no era así para el siglo XVI. Como plantea Sidney Mintz, «el uso de azúcar en Europa antes del siglo XVI (y, de hecho, aunque en menor medida, hasta el siglo XX), era primordialmente el de medicina y condimento, tan elevado era su precio y tan pequeña las cantidades en que solía consumírselo». ${ }^{71}$ Por ello se entiende que el capítulo inicie declarando que «aquesto del azúcar es una de las más ricas granjerías que en alguna provincia o reino puede haber, y en aquesta isla hay tanta e tan buena y de tan poco tiempo acá es así ejercida e adquirida». ${ }^{72}$

Traído por los europeos, el azúcar, había sido introducido al Mediterráneo por los árabes, y su expansión hacia el occidente estaba estrechamente vinculada a las expansiones ultramarinas de españoles y portugueses. En efecto, como indica Mintz ${ }^{73}$ las primeras plantaciones en Santo Domingo tuvieron que recurrir a un conocimiento especializado traído de las Canarias, antecedente directo de la explotación indiana. A ojos de Oviedo, la alta demanda del azúcar permitía cerrar un ciclo económico con el Viejo Mundo: «es de notar que hasta que hobo azucares en ella [en La Española], las naos tornaban vacías a España, e agora van cargadas della e con mayores fletes de los que para acá traen, e con más ganancia». ${ }^{74}$

\footnotetext{
70 Fernández de Oviedo, lib. VII, cap. VII, 1992, 1, 125.

71 Mintz, 2011, 231.

72 Fernández de Oviedo, lib. IV, cap. VII, 1992, 1, 106.

73 Mintz, 1996.

74 Fernández de Oviedo, lib. IV, cap. VIII, 1992, 1, 107.
} 


\section{Conclusión}

Ante cierta ineficacia de las categorías preconcebidas (provenientes de la obra de Plinio) con que Gonzalo Fernández de Oviedo se aproxima a la naturaleza del Nuevo Mundo, hemos planteado que una lectura detallada de las descripciones sobre la naturaleza indiana permite identificar una segunda trama clasificatoria, paralela a la anterior, que ubica a esta en una clave alimentaria.

Si la naturaleza del Nuevo Mundo se presenta «disforme» $\mathrm{y}$ «desemejante» a lo conocido por la sociedad europea, como un «mare magno e oculto», la entrada alimentaria permitirá a Fernández de Oviedo ubicarla en un código compartido de la tradición. No solo mediante relaciones miméticas, de analogías de sabores, colores y texturas, sino también como elementos consistentes con una organización dietética (médica y alimentaria) propia de la cultura tardo medieval. Así, la nueva trama de clasificación abre la posibilidad de reforzar en suelo indiano el imaginario de especiería que se había difuminado en los primeros años de la conquista.

Pero la entrada alimentaria no solo remite a la descripción de la naturaleza del Nuevo Mundo. En su intento por construir un corpus final del saber indiano, la Historia general y natural de las Indias - aunque asistemática - adquiere a momentos la dimensión de un verdadero compendio culinario. De los animales y plantas indianas, Fernández de Oviedo no solo comenta su sabor, sino que además enseña al lector como prepáralos, mezclando técnicas y procedimientos de ambos continentes, dando cuenta de una incipiente cocina mestiza.

En una línea establecida insistentemente por Daymond Turner no solo se ha planteado estar frente al primer cronista de Indias, sino que además Fernández de Oviedo es catalogado como el creador de la primera novela indiana, de la primera enciclopedia del Nuevo Mundo y, por tanto, el primer autor propiamente hispanoamericano. ${ }^{75}$ En este sentido, no sería osado agregar otro galardón a la lista y afirmar que fragmentos de su obra constituyen probablemente el primer recetario indiano que da cuenta no solo de las preparaciones indígenas sino también de una cocina mestiza.

Recibido el 8 de mayo de 2014 Aceptado el 16 de abril de 2015

75 Turner, 1964a, 1964b y 1976. 


\section{Bibliografía}

Álvarez, Enrique: «Plinio y Fernández de Oviedo I», Anales de Ciencias Naturales, I, Madrid, Instituto José de Acosta, CSIC, 1940, 40-61.

Álvarez, Enrique: «Plinio y Fernández de Oviedo II», Anales de Ciencias Naturales, II, Madrid, Instituto José de Acosta, CSIC, 1941, 1-23.

Álvarez, Enrique: «La Historia Natural en Fernández de Oviedo», Revista de Indias, XVII, 67-70, Madrid, 1957, 541-600.

Álvarez, Raquel: «La historia natural en tiempos del emperador Carlos V. La importancia de la conquista del Nuevo Mundo», Revista de Indias, LX, 218, Madrid, 2000, 13-31.

Aram, Bethany: Leyenda negra y leyendas doradas en la conquista americana, Madrid, Fundación Jorge Juan Marcial Pons, 2008.

Aram, Bethany: «From the Courts to the Court: History, Literature, and Litigation in the Spanish Atlantic World», Colonial Latin American Review, 21, 3, University of New Mexico, 2012, 343-364.

Ayala, María de la Luz: «La historia natural en el siglo XVI Oviedo, Acosta y Hernández», Estudios del Hombre, 20, Guadalajara, 2005,19-37.

Baraibar, Álvaro: «La naturaleza en el discurso indiano. La construcción de un espacio de experiencia americano», en Castany, Bernat (coord.), Tierras prometidas: de la colonia a la independencia, Barcelona, Bellaterra, 2011, 9-30.

Baraibar, Alvaro: «Las miradas de Gonzalo Fernández de Oviedo sobre la naturaleza del Nuevo Mundo», Estudos Ibero-Americanos, Porto Alegre, 40, 1, jan.-jun, 2014, 7-22.

Bolaños, Álvaro: «Panegírico y libelo del primer cronista de Indias, Gonzalo Fernández de Oviedo», Thesaurus: Boletín del instituto Caro y Cuervo, 45, 3, Bogotá, 1990, 577-649.

Bolaños, Álvaro: «La crónica de Indias de Fernández de Oviedo: ¿Historia de lo general y natural, u obra didáctica?», Revista de Estudios Hispánicos, 25, 3, Washington University in St. Louis, 1991, 15-33

Brandes, Stanley: «El misterio del maíz», en Janet Long (ed.), Conquista y comida, consecuencia del encuentro de dos mundos, Ciudad de México, Universidad Nacional Autónoma de México, 2011, 254-263.

Brokaw, Galen: «Ambivalence, Mimicry, and Stereotype in Fernandez de Oviedo's Historia general y natural de las Indias: Colonial Discourse and the Caribbean Areito», The New Centennial Review, 5, 3, Michigan, 2005, 143-165.

Carrillo, Jesús: «Naming Difference: The Politics of Naming in Fernández de Oviedo's Historia general y natural de las Indias», Science in Context, 16, 4, Cambridge, 2003, 489-504. 
Carrillo, Jesús: Naturaleza e imperio: la representación del mundo natural en la Historia general y natural de las Indias de Gonzalo Fernández de Oviedo, Madrid, Doce Calles/Fundación Carolina, 2004.

Coe, Sophie: Las primeras cocinas de América, Ciudad de México, Fondo de Cultura Económica, 2004.

Coello de la Rosa, Alexandre: «“Indios buenos?”, “¿Indios malos”, “¿Buenos cristianos?": la cara oscura de las Indias en Gonzalo Fernández de Oviedo y Valdés», Scripta Nova, Revista Electrónica de Geografía y Ciencias Sociales, V, 101, Barcelona, 2001. http://www.ub.edu/geocrit/sn-101.htm

Coello de la Rosa, Alexandre: «Representing the New World's Nature: Wonder and Exoticism in Gonzalo Fernández de Oviedo y Valdés», Historical Reflections/Réflexions Historiques, 28, 1, 2002a, 73-92.

Coello de la Rosa, Alexandre: De la naturaleza y el Nuevo Mundo: maravilla y exotismo en Gonzalo Fernández de Oviedo y Valdés (1478-1557), Madrid, Fundación Universitaria Española, 2002b.

Contreras, Jesús y García, Mabel: Alimentación y cultura. Perspectivas antropológicas, Barcelona, Ariel, 2005.

Egaña Rojas, Daniel Andrés: Escribir el Nuevo Mundo. La emergencia de la política en la obra de Gonzalo Fernández de Oviedo. Tesis de Doctorado en Ciencias Antropológicas, Universidad Autónoma Metropolitana, México, 2013. http://tesiuami.izt.uam.mx/uam/aspuam/ver_texto.php?recno=16100 $\&$ documento=UAMI16100.pdf

Fernández de Oviedo, Gonzalo: Historia general y natural de las Indias, Madrid, Atlas, 1992, 5 vols.

Fernández de Oviedo, Gonzalo: Sumario de la natural Historia de las Indias, Ciudad de México, Fondo de Cultura Económica, 1996.

Flandrin, Jean-Louis: «Condimentación, cocina y dietética durante los siglos XIV, XV, XVI», en Montanari, Massimo y Flandrin, Jean-Louis (dirs.), Historia de la alimentación, Gijón, Ediciones Trea, 2011, 625-645.

Foucault, Michel: Las palabras y las cosas. Una arqueología de las ciencias humanas, Buenos Aires, Siglo XXI Editores, 2003.

Gerbi, Antonello: La naturaleza de las Indias Nuevas: de Cristobal Colon a Gonzalo Fernández de Oviedo, Ciudad de México, Fondo de Cultura Económica, 1992.

Myers, Kathleen: «History, Truth and Dialogue: Fernández de Oviedo's Historia general y natural de las Indias (Bk XXXIII, Ch LIV)», Hispania, 73, 3, 1990, 614-25.

Myers, Kathleen: Fernández de Oviedo's Chronicle of America. A New History for a New World, Austin, University of Texas Press, 2007.

Mintz, Sidney: Dulzura y poder, el lugar del azúcar en la historia moderna, Ciudad de México, Siglo XXI Editores, 1996. 
Mintz, Sidney: «El dulce intruso: el azúcar en el Nuevo Mundo», en Long, Janet (ed.), Conquista y comida, consecuencia del encuentro de dos mundos, Ciudad de México, Universidad Nacional Autónoma de México, 2011, 227-235.

Montanari, Massimo: El queso con las peras. La historia de un refrán, Gijón, Ediciones Trea, 2018.

Otte, Enrique: «Una carta inédita de Gonzalo Fernández de Oviedo», Revista de Indias, XVI, 65, Madrid, 1956, 437-58.

Paden, Jeremy: «The Iguana and the Barrel of Mud: Memory, Natural History, and Hermeneutics in Oviedo's Sumario de la natural historia de las Indias», Colonial Latin American Review, 16, 2, 2007, 203-226.

Palerm, Ángel: Historia de la etnología. I Los precursores, Ciudad de México, Universidad Iberoamericana, 2006.

Peña y Cámara, José de la: «Contribuciones documentales para una biografía de Gonzalo Fernández de Oviedo», Revista de Indias, XVII, 69-70, Madrid, 1957, 603-705.

Regueiro, Antonio: «La flora americana en la España del siglo XVI», en Solano, Francisco de y Pino, Fermín del (eds.), América y la España del siglo XVI. Homenaje a Gonzalo Fernández de Oviedo cronista de Indias en el V centenario de su nacimiento, Madrid, Instituto Fernández de Oviedo, 1983, I, 205-217.

Ríos, José Amador de los: «Vida y escritos de Gonzalo Fernández de Oviedo», en Historia general y natural de las Indias de Gonzalo Fernández de Oviedo, Madrid, Academia de la Historia, 1851, I, IX-CVII.

Rodilla, José María: «Bestiarios del Nuevo Mundo: maravillas de Dios o engendros del demonio», Rilce. Revista de filología hispana, 23, 1, Navarra, 2007, 195-205.

Turner, Daymond: «Gonzalo Fernández de Oviedo's Historia general y natural: First American Encyclopaedia», Journal of Inter-American Studies, VI, 2, Gainesville, 1964a, 267-274.

Turner, Daymond: «Oviedo's Claribalte: the First American Novel», Romance Notes, VI, 1, Chapel Hill, 1964b, 65-68.

Turner, Daymond: «Gonzalo Fernández de Oviedo y Valdés: First SpanishAmerican Author», en Charles L. Nelson (ed.), Studies in Language and Literature: The Proceedings of the 23rd Mountain Interstate Foreign Language Conference, Richmond, Easter Kentucky University, 1976, 557563.

Vázquez, Josefina Zoraida: «El indio Americano y su circunstancia en la obra de Fernández de Oviedo», Revista de Indias, XVII, 69-70, Madrid, 1957, 483-520. 